Signal \& Image Processing : An International Journal (SIPIJ) Vol.6, No.4, August 2015

\title{
AN IMPROVED ANC SYSTEM WITH APPLICATION TO SPEECH COMMUNICATION IN NOISY ENVIRONMENT
}

\author{
Narayanan N.K. ${ }^{1}$ and Sivadasan Kottayi ${ }^{2}$ \\ ${ }^{1}$ Information Technology Department, Kannur University, Kannur 670567, India. \\ ${ }^{2}$ Electronics \& Instrumentation Engineering Technology Department, \\ Yanbu Industrial College, Yanbu, Saudi Arabia.
}

\begin{abstract}
A new two stage FXNLMS algorithm based ANC system with secondary path modelling was proposed in [15].Performance analysis of this system with real signals has been carried out using computer simulation. Simulation studies showed that the system after trained with WGN can be successfully used for reducing wide sense broad band noise.This paper also explores the intelligibility of speech signals in the quiet zone created by the ANC system. The utterances of phonetically balanced Harvard sentences with different SNRs are propagated through the quiet zone and the intelligibility of the resultant speech signal is measured using MOS (Mean Opinion Score) test. Encouraging results are obtained which indicates that the two stage FXNLMS algorithm based ANC system can find application in mobile phone communication.
\end{abstract}

\section{KEYWORDS}

Active noise control, speech intelligibility, FxLMS algorithm, online secondary path modelling, Adaptive systems, DSP applications

\section{INTRODUCTION}

Now-a-days Active Noise Control is emerging as an effective technique for nullifying noise [1, 2]. High frequency noise can be cancelled through conventional passive methods [3], which employ heavy barriers to block the transmission of sound and also use certain acoustic materials to absorb sound energy. However, this technique is less effective in low frequency $(<500 \mathrm{~Hz})$. To stop such kind of noise one needs to have barriers with large width which are expensive and often hard to implement. In mobile telephony, it is difficult to attend incoming telephone calls in a noisy environment. At the same time while making a call in a noisy environment, surrounding noise propagates along with speech signal to the destination. In such situations, conventional passive techniques cannot be applied, but noise can be eliminated electronically through ANC.

\section{EXISTING FEED-FORWARD ANC SYSTEMS}

Feed forward ANC system is realized in system identification configuration of adaptive filter $[2,4]$. In system identification configuration, the output of adaptive filter is directly connected to 
Signal \& Image Processing : An International Journal (SIPIJ) Vol.6, No.4, August 2015

summation point. However, in ANC system the output of adaptive controller passes through various subsystems, namely, D/A converter, low pass filter, loudspeaker, acoustic path between loudspeaker and error microphone, error microphone, A/D converter etc. Transfer functions of all these subsystems are collectively called secondary path transfer function $S(z)$ which is to be compensated for optimum performance. To tackle this problem FxLMS algorithm has been proposed [4, 5]. In FxLMS algorithm $S(z)$ has to be estimated. Various methods of online modelling of $S(z)$ are reported in literature $[6,7,8,9,10,11,12,13,14,15]$. The ANC system proposed in [13] modelled secondary path as delay block plus random noise. However, modelling $S(z)$ as delay block plus random noise is an over simplified approach as secondary path consists of numerous real sub-systems whose transfer functions are complex in nature. Widrow and Stearns proposed an ANC system [5] where, secondary path modelling is done by exciting $S(z)$ using the output of the noise control filter, which results in a signal depended solution. In [6] zero mean random noise $v(n)$ with variance 1 is used as an exciting signal for modelling $S(z)$ which is widely accepted. However, the random noise $v(n)$ used for exciting $S(z)$ interfere noise control filter and the primary noise $x(n)$ interfere the modeling of $S(z)$. The ANC system proposed in [7], an additional adaptive filter $\hat{B}(z)$ with $x(n)$ as reference signal is introduced for cancelling the undesired component of the error signal due to the primary signal for improving the modelling of $S(z)$. But the interference of the undesired component of the error signal due to $v(n)$ adversely affects the convergence of noise control filter $W(z)$. Zhang et al [8] used three cross updated adaptive filters, which improves the performance of noise control filter as well as modelling of $\mathrm{S}(\mathrm{z})$. However, the drawback of this system is that the error signal $g(n)$ using as desired signal for estimating $\hat{S}(z)$ is not completely free from primary noise. All the above outlined systems use FxLMS algorithm for updating active noise control filter and LMS algorithm for secondary path modelling. However, other existing systems $[9,10,11,12$, 14] use modified FxLMS (MFxLMS) algorithm for noise control filter and variable step size (VSS) LMS algorithm for the adaptation of secondary path modelling filter make the system complex.

The two stage FxNLMS algorithm based ANC system proposed in [15] used FxNLMS algorithm for active noise control filters and NLMS algorithm for secondary path modelling filters, which does not have any computational complexity. Simulation studies reported in [15] shows superior performance of the system compare to similar type of systems. This paper evaluates the performance of this system by computer simulation using various real noise signals and also tests speech intelligibility in the quiet zone created by the system.

\section{Perforance of The Two Stage FXnLMS BaSed ANC System WITH REAL NOISES}

The system proposed in [15] is first trained by tuning the step sizes of all its adaptive filters using WGN signal of $1 \mathrm{dBW}$ with normalized amplitude of \pm 1 unit as primary source. This trained system is tested by simulation with normalized real noises collected from transport vehicles (Car, bus, train, aircraft etc.), offices (due to Air-conditioners, fans etc.) and kitchen of a house (due to exhaust fan) as primary sources. 
Signal \& Image Processing : An International Journal (SIPIJ) Vol.6, No.4, August 2015

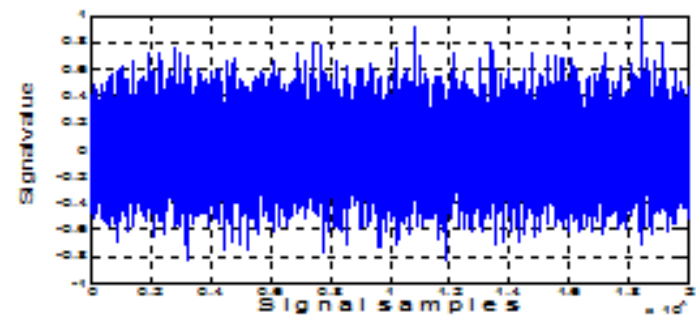

Fig 1(a). WGN as primary signal

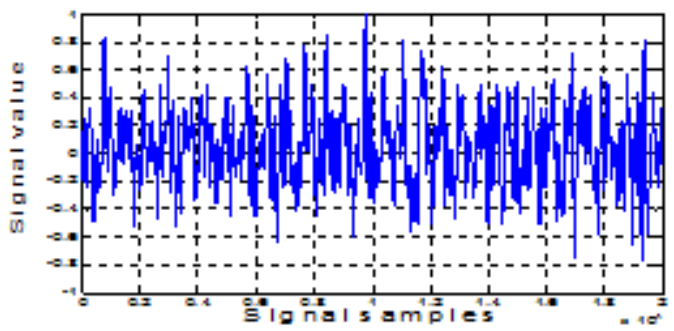

Fig 1(c). Noise collected from a car as primary signal

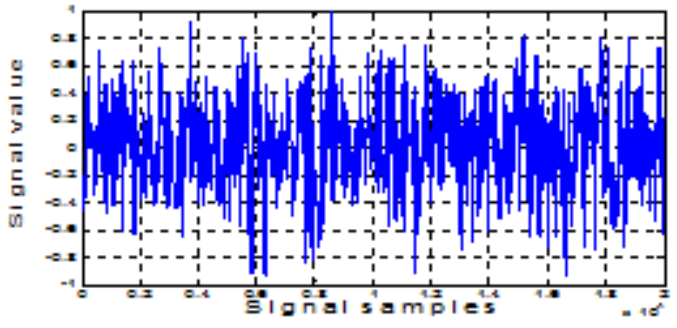

Fig 1(e). Noise collected from a bus as primary signal

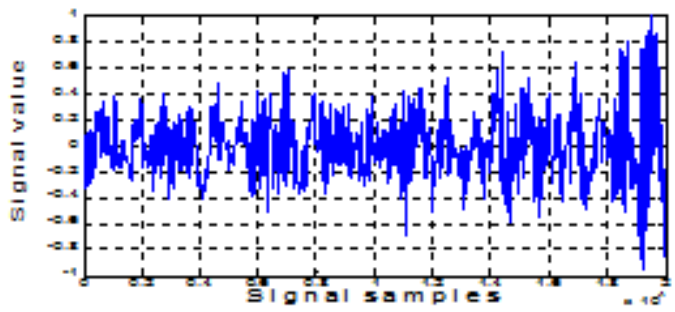

Fig 1(g). Noise collected from a train as primary signal

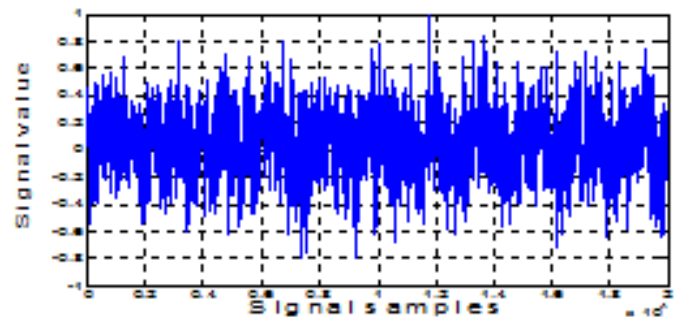

Fig 1(i). Noise collected from an aircraft as primary signal

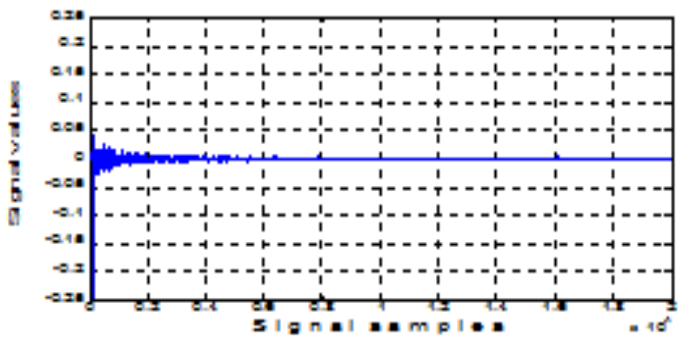

Fig 1(b). Residual noise when WGN is the primary signal

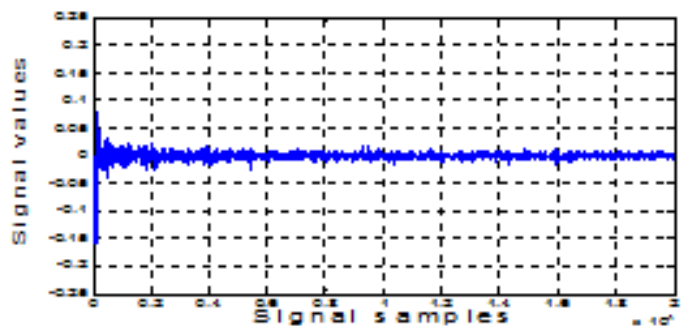

Fig 1(d). Residual noise in the case of a car noise

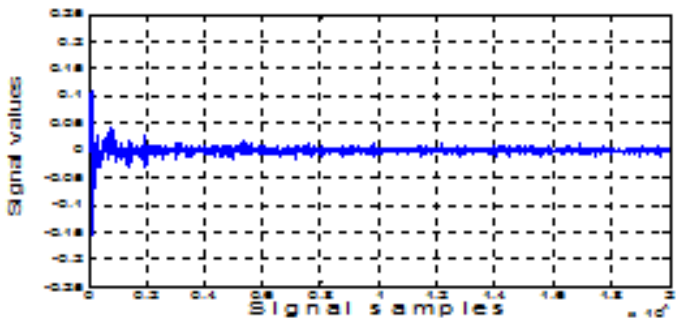

Fig 1(f). Residual noise in the case of a bus noise

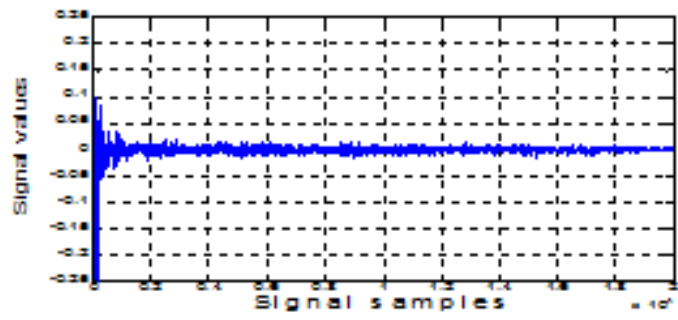

Fig 1(h). Residual noise in the case of train noise

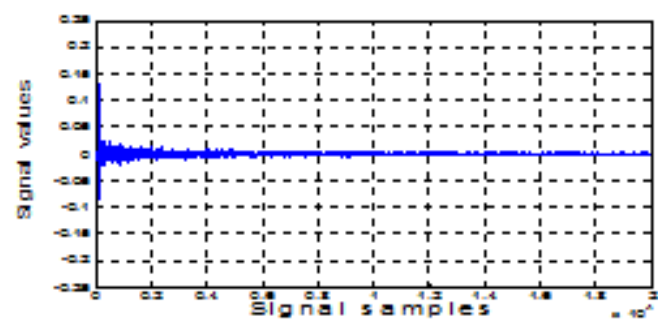

Fig 1(j). Residual noise in the case of an aircraft noise 
Signal \& Image Processing : An International Journal (SIPIJ) Vol.6, No.4, August 2015

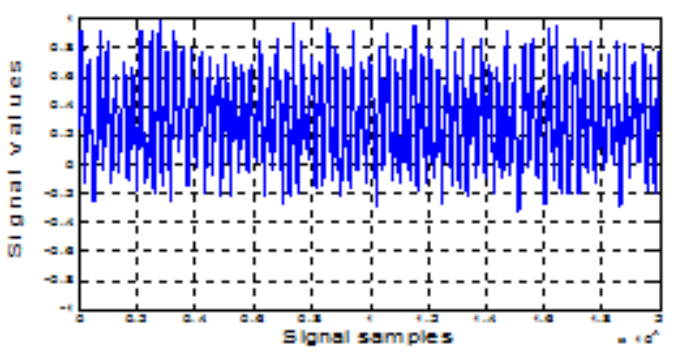

Fig 1(k) Office noise due to the working of air conditioner

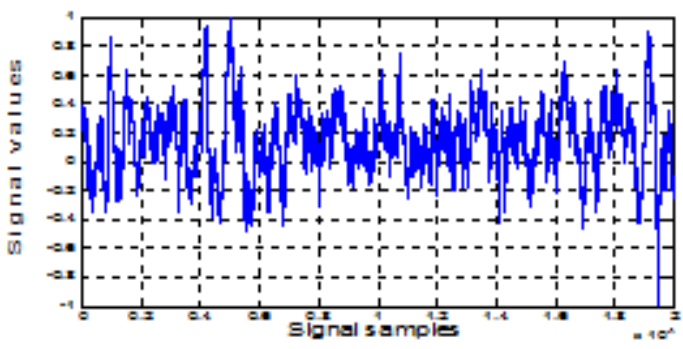

Fig 1(m) Office noise due to the working of fans

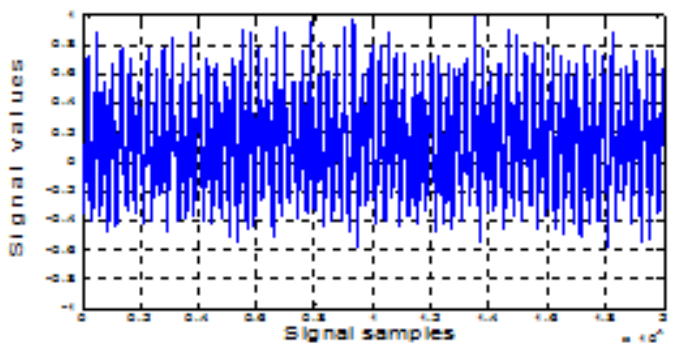

Fig 1(o) House noise due to the working of kitchen exhaust fan

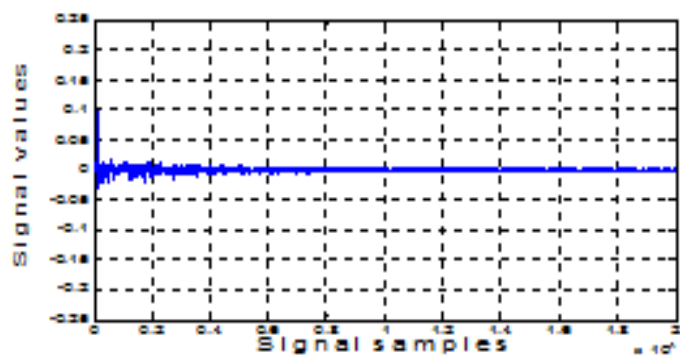

Fig 1(1) The residual noise in the case of Air-conditioner noise

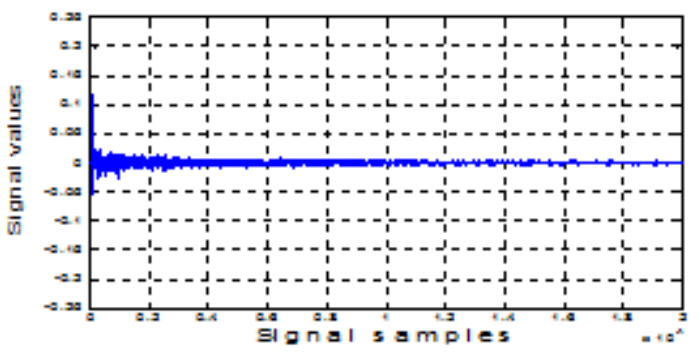

Fig 1(n) The residual noise in the case of fan noise

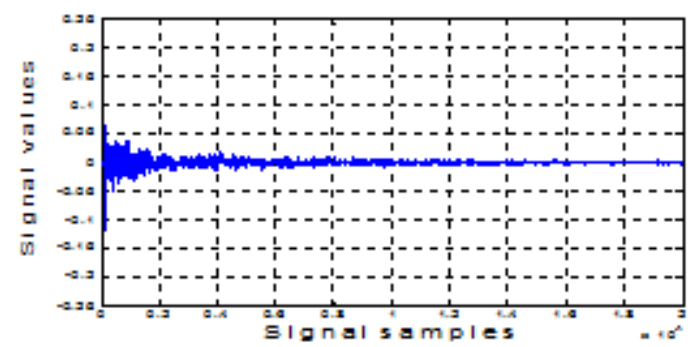

Fig 1(p) The residual noise in the case of kitchen exhaust fan

Fig $1(a-p)$ Simulation results of the system with real signals

The performance evaluation of the system is done by calculating the relative average power $(\mathrm{dB})$ of error output (residual noise), $e(n)$ to the primary signal $x(n)$ using the formula

$$
10 \log \left[\left\{\frac{\sum_{n=1}^{L}\{e(n)\}^{2}}{L}\right\} /\left\{\frac{\sum_{n=1}^{L}\left\{\{x(n)\}^{2}\right\}}{L}\right\}\right]
$$

where $\mathrm{L}$ is the maximum number of samples. The order of all the active filters is kept as 64 and the numbers of samples of the signals are taken as 20000. Fig. 1(a) shows the WGN signal and Fig. 1(b) is the residual output of the system when WGN is the primary signal. Simulation results while using noises collected from transport vehicles as primary sources are given in Fig. 1(c) to Fig. 1(j). Simulation results in the case of office noise and house noise as primary sources are shown in Fig. 1(k) to Fig. 1(p). The reduced output power in each case is calculated using the equation (1) and is given in Table 1. 
Signal \& Image Processing : An International Journal (SIPIJ) Vol.6, No.4, August 2015

Alternatively, without training the system with WGN, the ANC system is simulated by considering each signal mentioned above as primary signal and step sizes of all its adaptive filters tuned and optimized separately. Such signal-depended system for each case has been tested separately. The noise reduction at the output is found out using equation (1) for each case and is also given in Table 1. On the basis of the experiments, from Table 1 it is evident that noise reduction at the output is almost same in both approaches which is an important result that the two stage FxNLMS based ANC system trained using WGN can be used in any practical widesense broad band noise environment for noise elimination.

Table 1: Performance of the system with real signals

\begin{tabular}{|c|c|c|c|}
\hline \multirow[b]{2}{*}{ S. No } & \multirow[b]{2}{*}{ Primary source } & \multicolumn{2}{|c|}{$\begin{array}{l}\text { Noise reduction at the } \\
\text { output in } \mathrm{dB}\end{array}$} \\
\hline & & $\begin{array}{l}\text { The } \\
\text { system } \\
\text { after } \\
\text { trained } \\
\text { using } \\
\text { WGN }\end{array}$ & $\begin{array}{l}\text { The system } \\
\text { tuned for } \\
\text { individual } \\
\text { real noise } \\
\text { separately }\end{array}$ \\
\hline $\mathbf{A}$ & $\begin{array}{l}\text { With noises from Transport } \\
\text { Vehicles }\end{array}$ & & \\
\hline 1 & Car noise & -32.3 & -34 \\
\hline 2 & Bus noise & -35.38 & -36 \\
\hline 3 & Train noise & -35.68 & -35 \\
\hline 4 & Aircraft noise & -34.56 & -36.9 \\
\hline B & With Office noise & & \\
\hline 1 & $\begin{array}{l}\text { Office noise due to air- } \\
\text { conditioners }\end{array}$ & -41.11 & -41.7 \\
\hline 2 & Office noise due to fan noise & -38.32 & -38.5 \\
\hline C & With house noise & & \\
\hline 1 & Kitchen exhaust fan noise & -34.8 & -33.7 \\
\hline
\end{tabular}

\section{TESTING OF SPEECH INTELLIGIBILITY}

In this section we explore the intelligibility of speech signal propagated through the quiet zone created by the ANC system. The two stage FxNLMS algorithm based ANC system together with speech signal is shown in Fig. 2. The physical arrangement of the system can be seen in [15]. When speech signal propagates through the quiet zone created by the ANC system between loudspeakers and error microphones, slight degradation of lower frequencies of the speech signal takes place as the proposed ANC system is designed for cancelling low frequency noise ( $\leq$ $500 \mathrm{~Hz})$.

A set of phonetically balanced utterances of Harvard list of sentences (1. An icy wind racked the beach, 2. The pipe began to rust while new, 3. Cats and dogs hate each the other, 4. Oak is strong and also gives shade, 5. Thieves who rob friends deserve jail, 6. Open the crate but do not break the glass, 7. Add the sum to the product of these three, 8. Joe brought a young girl, 9. A lathe is a big tool.) mixed with WGN with different SNRs (-10dB to $40 \mathrm{~dB})$ and propagated in the quiet zone created by the ANC system. This signal is denoted as $z(n)$ in Fig. 2. The primary signal $x(n)$ used in this experiment is WGN. In simulation, intelligibility of speech signal is 
Signal \& Image Processing : An International Journal (SIPIJ) Vol.6, No.4, August 2015

measured using MOS (Mean Opinion Score) test by hearing the output of the system by 30 different people and average score is recorded for each SNR value.

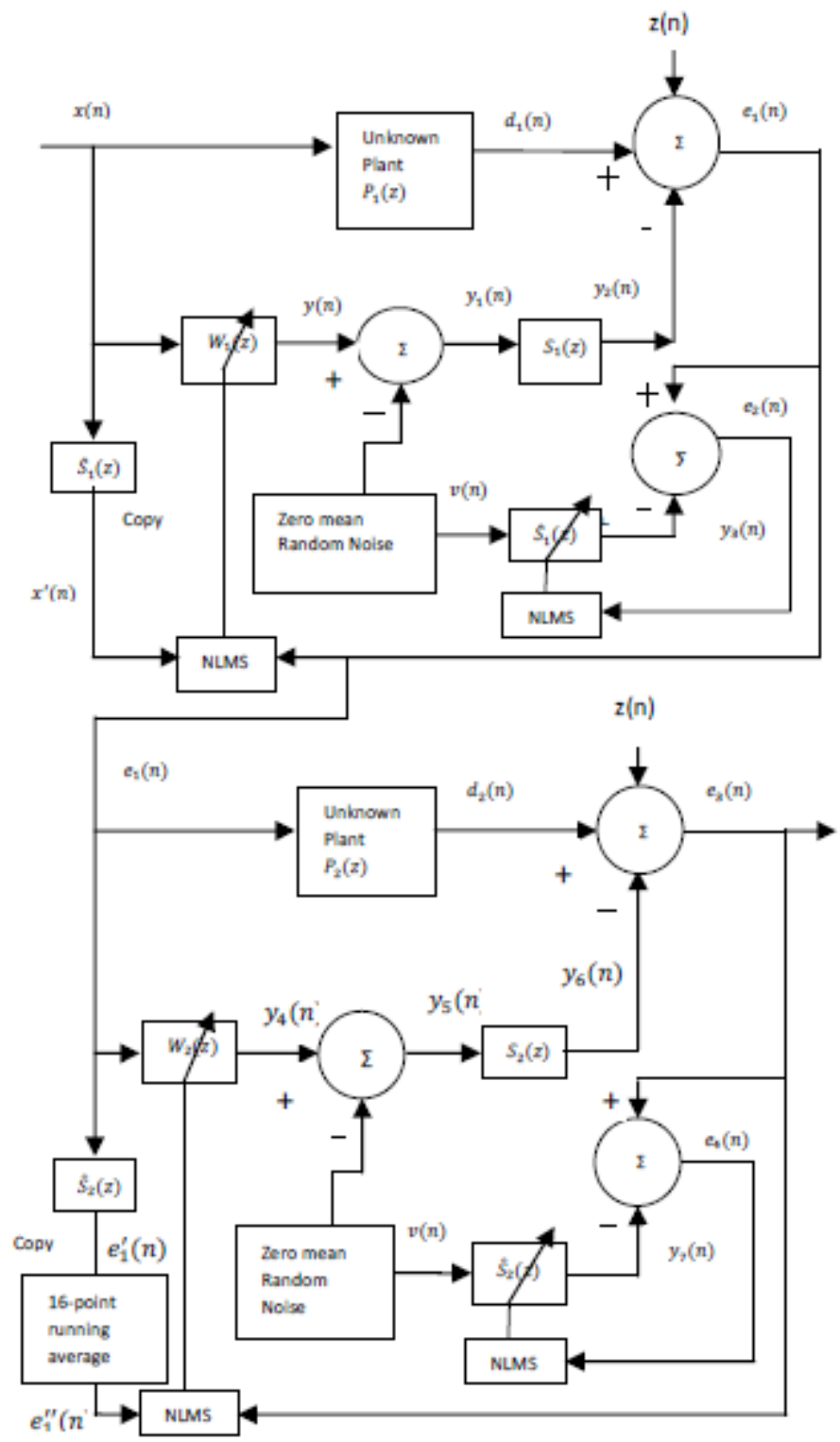

Fig. 2. Two stage FxNLMS algorithm based ANC system together with speech signal z(n)

The speech inputs and outputs of the system are plotted in Fig. 3(a-r) by taking an example of the utterance, "An icy wind racked the beach". It is tested on the proposed system by changing SNR from $-10 \mathrm{~dB}$ to $40 \mathrm{~dB}$. A Clean utterance of the said sentence without corrupted with noise is also tested. Tests are also done using the utterances of all other Harvard list of sentences mentioned above. The intelligibility of speech signal is measured by finding the average of the results of the MOS tests of all the sentences in different SNRs and is given in Table 2. 
Signal \& Image Processing : An International Journal (SIPIJ) Vol.6, No.4, August 2015

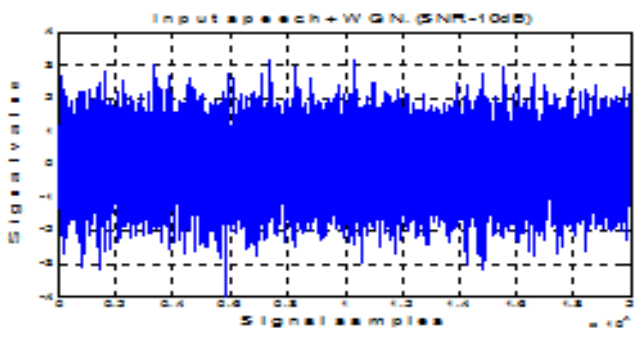

Fig. 3(a)

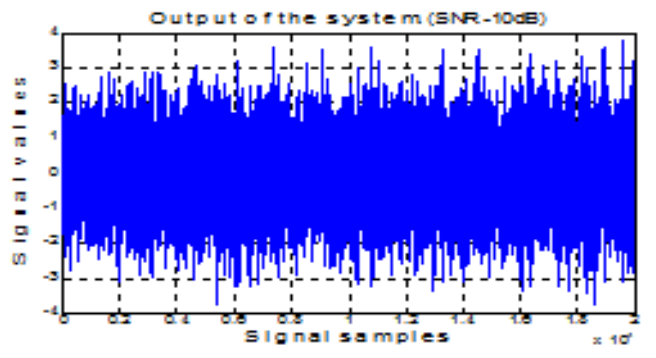

Fig. 3(b)

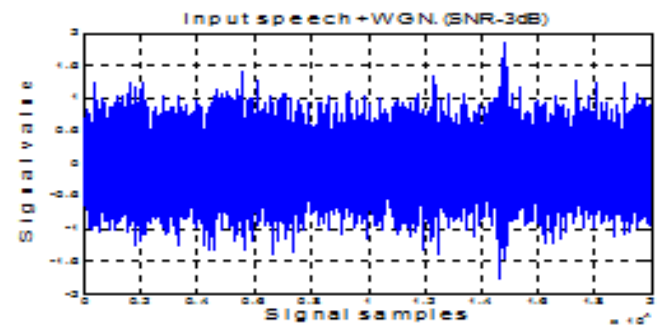

Fig. 3(c)

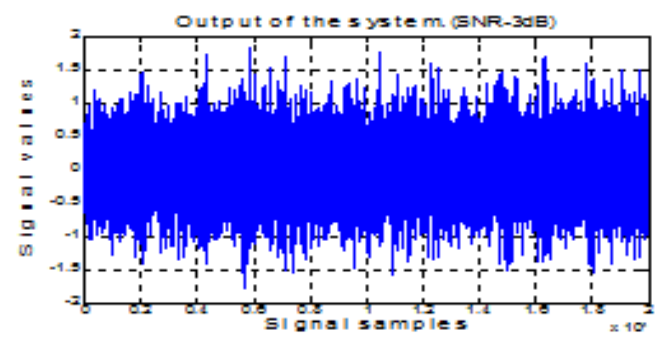

Fig. 3(d)

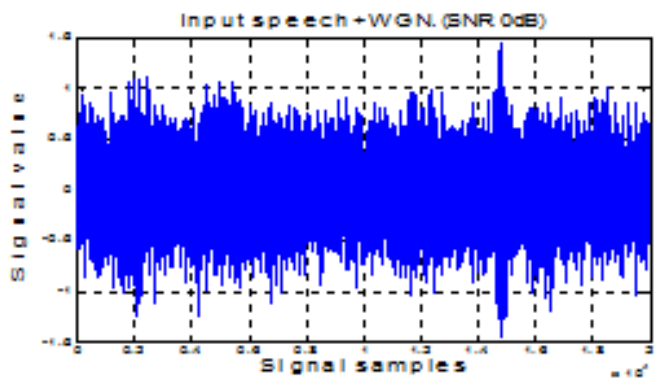

Fig. 3(e)

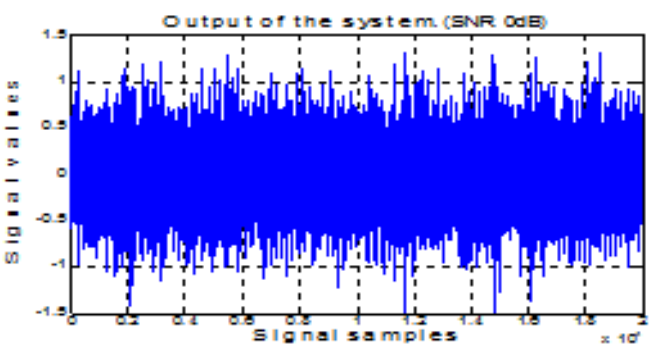

Fig. 3(f)

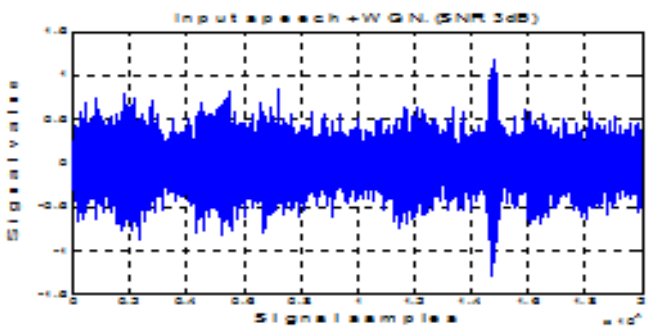

Fig. 3(g)

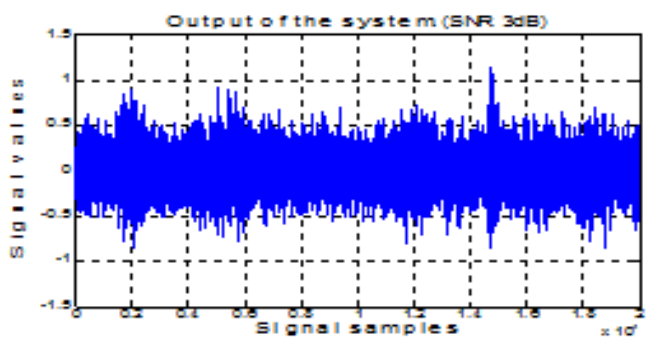

Fig. 3(h)

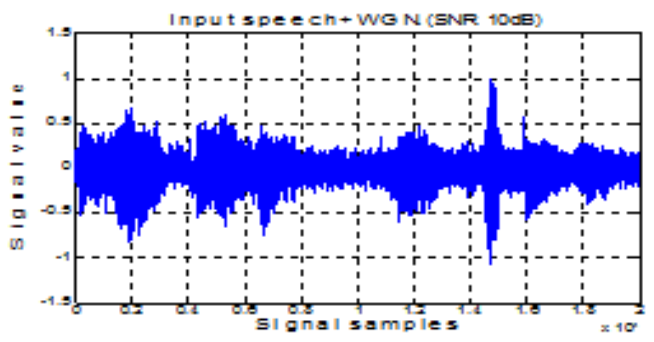

Fig. 3(i)

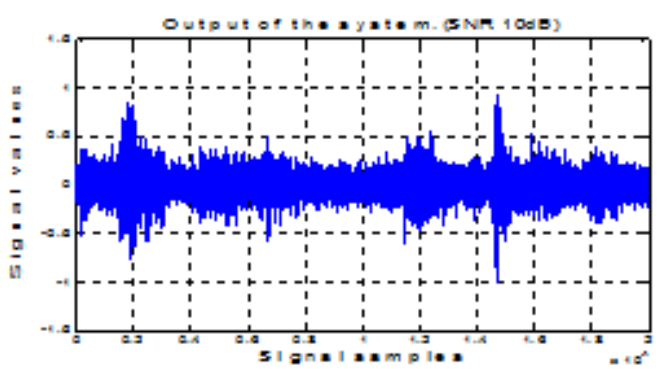

Fig. 3(j) 
Signal \& Image Processing : An International Journal (SIPIJ) Vol.6, No.4, August 2015

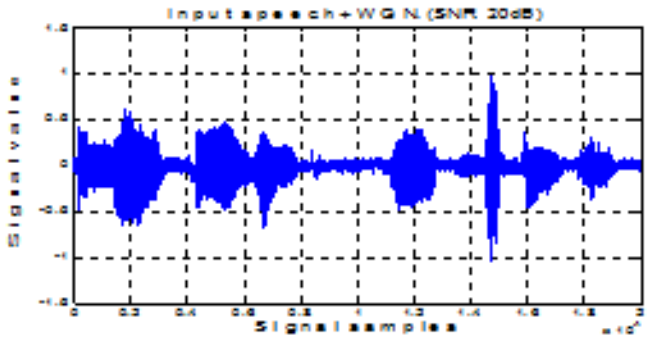

Fig. 3(k)

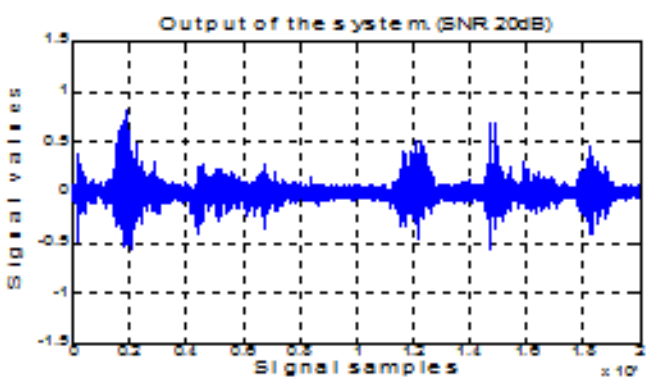

Fig. 3(1)

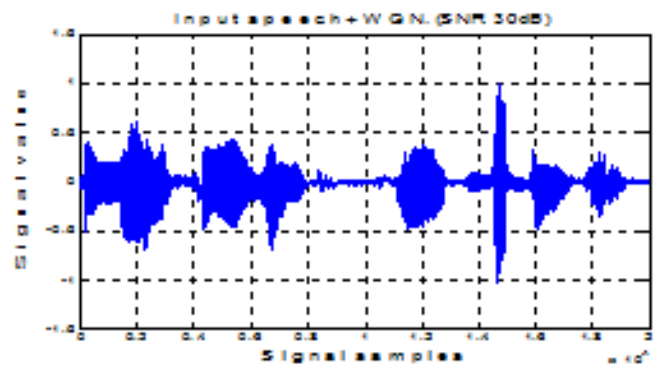

Fig. 3(m)

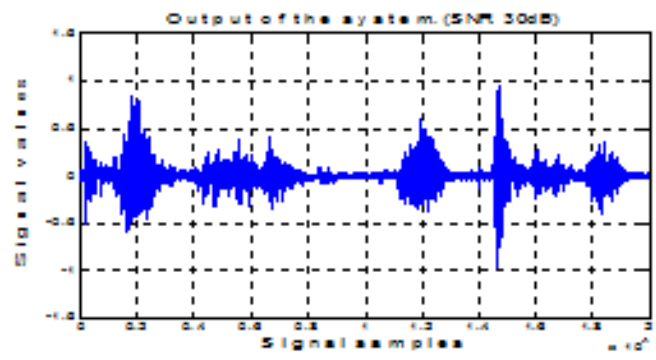

Fig. 3(n)

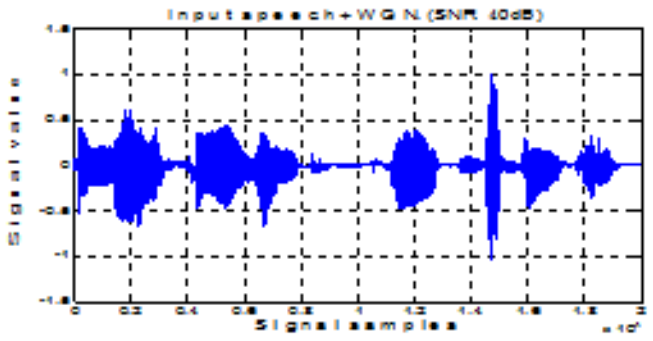

Fig. 3(o)

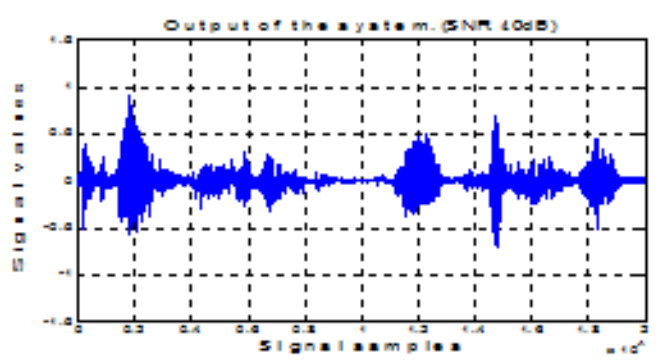

Fig. 3(p)

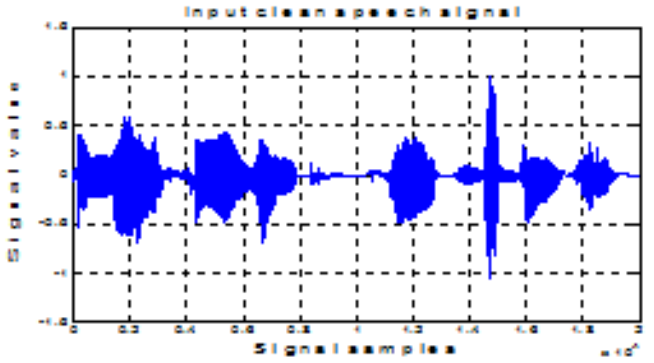

Fig. 3(q)

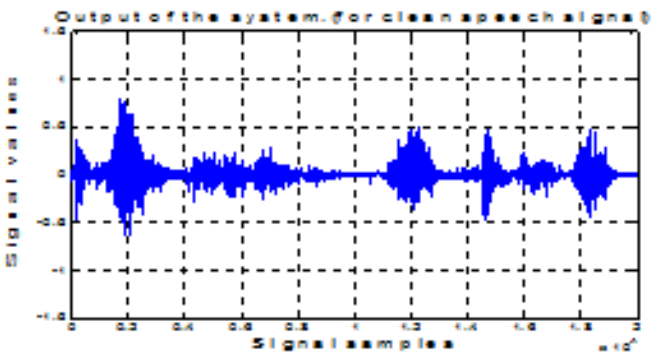

Fig. 3(r)

Fig.3 (a-r) Test results (Noisy spech inputs and outputs of the system) using the utterance "An icy wind racked the beach" for different SNRs

From Table 2 it is evident that the speech signals whose SNR is above $3 \mathrm{~dB}$ do not lose their intelligibility. The above mentioned tests simulate a scenario such that if an ANC system is built within a mobile phone and creates a quiet zone around the mobile phone user, the mobile phone user's speech as well as the speech signals come out from the received calls retains their intelligibility. Therefore the two stage FxNLMS algorithm based ANC system can find application in mobile phones. 
Signal \& Image Processing : An International Journal (SIPIJ) Vol.6, No.4, August 2015

Table 2 Average MOS result of the utterances of harvad list of sentences

\begin{tabular}{|c|c|c|}
\hline $\begin{array}{c}\text { Speech signal } \\
\text { with different } \\
\text { SNR } \\
\text { dB }\end{array}$ & $\begin{array}{c}\text { Average } \\
\text { Mean } \\
\text { observation } \\
\text { Score (MOS) }\end{array}$ & $\begin{array}{c}\text { Intelligibility in } \\
\text { terms of Grade }\end{array}$ \\
\hline-10 & 1.0 & bad \\
-3 & 1.0 & bad \\
0 & 1.5 & poor \\
3 & 2.5 & fair \\
10 & 2.8 & fair \\
20 & 3.4 & good \\
30 & 3.7 & good \\
40 & 4.5 & Excellent \\
Clean speech & 4.5 & Excellent \\
\hline
\end{tabular}

\section{CONCLUSION}

This paper demonstrates performance evaluation of the two stage FxNLMS based ANC system under different noise environments. Simulation studies successfully revealed that this ANC structure trained with WGN can be used for noise cancellation of wide-sense broad band noise such as noise from transport vehicles, noise from offices etc. The paper also exposes by simulation experiments that speech signals retain their intelligibility in the quiet zone created by the ANC system. Hence, the system can be used in mobile phones for creating a quiet zone around a mobile user.

\section{REFERENCES}

[1] Kuo, S. M. \& Morgan, D. R. (1996) Active Noise Control Systems - Algorithms and DSP implementations. John Wiley \& Sons Inc., New York.

[2] Hansen, C. H. (2001) Understanding active noise cancellation. Spon Press, London.

[3] Beranek, L. L. \& Ver, I. L. (1992) Noise and Vibration Control Engineering: Principles and Applications. Wiley, New York.

[4] Morgan, D.R. (1980). "An analysis of multiple correlation cancellation loops with a filter in the auxiliary path", IEEE Trans. Acoustic. Speech, Signal Processing, vol. ASSP - 28, pp454 - 467.

[5] Widrow, B. \& Stearns, S.D. (1985) Adaptive Signal Processing. N.J: Prentice-Hall, Englewood Cliffs.

[6] Eriksson, L.J. and Allie, M.C. (1989) "Use of random noise for on-line transducer modelling in an adaptive active attenuation system", Journal of Acoust. Society of Amrica, Vol 85, pp797-802.

[7] Bao, C. P., Sas \& Van, B. H. (1993). "Adaptive active control of noise in 3D reverberant enclosures", Journal of Sound and Vibration, vol 161, no. 3, pp501-514.

[8] Zhang, M. Lan, H., \& Ser, W. (2001) Cross-updated active noise control system with online secondary path modelling. IEEE Trans. Speech Audio Process., vol. 9, no. 5, pp598 - 602.

[9] Akhtar, M.T., Abe, M. \& Kawamata, M., (2005) "A new structure for feed forward active noise control systems with improved online secondary path modelling", IEEE Transaction on Speech and Audio Processing, vol. 13, No.5, pp1082-1088.

[10] Akhtar, M.T., Abe, M. \& Kawamata, M., (2006) "A new variable step size LMS algorithm based method for improved online secondary path modelling in active noise control systems", IEEE Transactions on Audio, speech and Language processing, vol. 14, No.2, pp720 - 726. 
Signal \& Image Processing : An International Journal (SIPIJ) Vol.6, No.4, August 2015

[11] Alberto, C. \& Silvia, M. (2008) "Optimal variable step-size NLMS algorithms with auxiliary noise power scheduling for feedforward active noise control", IEEE Trans. Audio, Speech and Lang Process, vol. 16, no. 8, pp1383-1395.

[12] Pooya, D. and Hamid, H. (2008) "A variable step-size FxLMS algorithm for feedforward active noise control system based on a new online secondary path modelling technique", Proceedings of the International conference on computer systems \& applications, AICCSA 08, pp74-81.

[13] Sivadasan K. and Narayanan N. K. (2011), "Active Noise Control of Passenger Train Cabin with secondary path modelled as delay block plus random noise", Proceedings of the international conference in New Paradigms in Electronics \& Information Technologies (PEIT '011), Alexandria, Egypt.

[14] Shakeel, A. Akhtar, M.T. Zhang, Xi. (2013). "Robust Auxiliary-Noise-Power Scheduling in ANC with online secondary path modelling", IEEE Transactions Audio, Speech and Language Processing, Vol. 21, No. 4, pp749-760.

[15] Sivadasan, K. \& Narayanan, N.K.(2013), "A new online secondary path modelling for feedforward ANC System", International Journal of Electronics Letters, Taylor \& Francis, U.K., Vol.1, issue 4, pp179- 188 .

\section{AUTHORS}

\section{N. K. NARAYANAN}

N.K. Narayanan is currently working as Senior Professor in the School of Information Science \& Technology, Kannur University, Karala, India. He has served as Principal, College of Engineerng, Vadakara, Kerala, India during 2008 2010. He earned a Ph.D in speech signal processing from Department of Electronics, CUSAT, Kerala, India in 1990. He has published more than hundred research papers in national \& international journals in the area of Speech processing, Image processing, Neural networks, Bioinformatics and Active Noise Control Systems. He is a fellow of Acoustical Society of India

\section{SIVADASAN KOTTAYI}

Sivadasan Kottayi had his M.Tech (Electronics \& Communication Systems) from CUSAT, Kerala, India in 1981. He served BITS, Pilani; CEERI, Pilani; NERIST, Itanagar; JNAU, Jabalpur; Defence University, Ethiopia; Arbaminch University, Ethiopa in different capacities. Currently he is working as a faculty member of EIET Department of Yanbu Industrial College, under Royal Commission Yanbu Colleges \& Institutions (RCYCI), Saudi Arabia. He had to his credit two patents and many research publications in national and international Journals in the area of Instrumentation, Industrial Process Control, Microprocessor/ microcontroller applications, Active noise control and Digital Signal Processing. He is a fellow of Institution of Electronics and Telecommunication Engineers (India)
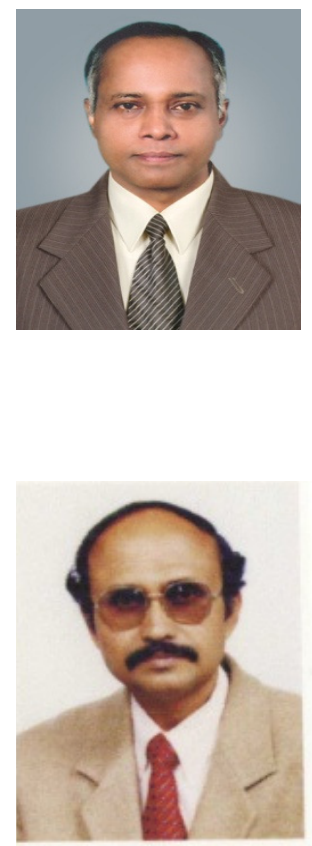